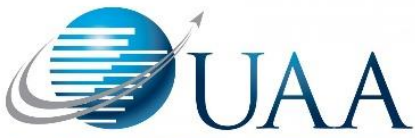

Collegiate Aviation Review

International

UNIVERSITY AVIATION

ASSOCIATION

\title{
How Air Traffic Control Intervention Effects Altitude Deviations on Optimized Profile Descent Arrivals
}

\author{
Donald E. Lyle \\ Wind Quest Trajectories, LLC
}

\begin{abstract}
U.S. National Airspace System modernization began with the publication of the Next Generation Air Transportation System Integrated Plan (NextGen) in 2004 to accommodate forecasted air travel demand increases in the United States. This framework proposed an integrated approach to safety, environmental sustainability, reduced fuel burn, and increased airspace and airport capacity by using automated capabilities. One of these capabilities, the Optimized Profile Descent (OPD) is an automated procedure created to link the en route phase of flight with the terminal area within the context of NextGen goals. This type of automated procedure was developed during the NextGen short phase (2004-2012) for both air traffic control and aircraft but continue to be used in a non-integrated manner. It is the confluence of incompatible automated and manual air traffic management techniques that produce a favorable location for an altitude deviation. The purpose of this study is to determine the effect of air traffic control intervention on altitude deviations reported during optimized profile descent arrival procedures in the U.S. National Airspace System from January 1, 2012 to January 1, 2018. Examination of aviation safety reports from this time period showed that air traffic control intervention did affect altitude deviations, specifically in the areas of aircrew error, communication error, and equipment malfunction or limitation. This analysis also demonstrated the failure of the altitude deviation rate to return to normal historic levels after the introduction of NextGen procedures, making altitude deviation a leading safety indicator for the U.S. National Airspace System.
\end{abstract}

\section{Recommended Citation:}

Lyle, D.E. (2021). How Air Traffic Control Intervention Effects Altitude Deviations on Optimized Profile

Descent Arrivals. Collegiate Aviation Review International, 39(1), 111-122. Retrieved from

https://ojs.library.okstate.edu/osu/index.php/CARI/article/view/8217/7587 
The purpose of this study was to determine the effect of air traffic control intervention on altitude deviations reported during optimized profile descent arrival procedures in the U.S. National Airspace System (NAS) from January 1, 2012 to January 1, 2018. The Next Generation Air Transportation System Integrated Plan (NextGen) (U.S. Department of Transportation [U.S. DOT], 2004) establishes a framework for safety, environmental sustainability, fuel burn and emissions reduction, and increased airport and airspace capacity through the greater use of integrated, complimentary automated capabilities. The literature to date has dealt with concepts and procedures to be used in an increasingly automated NextGen system, with few studies investigating the potential safety implications of using NextGen in a non-integrated fashion. When NextGen is not used as intended, its economic and environmental benefits are not realized, and the safety elements designed into its procedures are bypassed. For example, a single U.S. major airline flew 4,000 flights per day in 2018 (Southwest Airlines, 2018). If one half of those daily flights $(2,000)$ flew an automated optimized profile descent (OPD) arrival uninterrupted, it would save the airline $\$ 25$ million (US) per year in fuel costs and reduce its carbon emissions by 250 million pounds annually with a jet fuel price of \$2 (US) per gallon (Conklin \& de Decker, 2021; Lyle, 2020). These economic and environmental benefits are in addition to the increased safety of greater predictability and reduced uncertainty offered by automated NextGen procedures.

Why Air Traffic Control Intervention is Disruptive. An altitude deviation is defined as a departure "...from the assigned altitude (or flight level) equal to or greater than 300 feet....and may result in substantial loss of aircraft vertical or horizontal separation, which could cause a mid-air collision" (Flight Safety Foundation, 2000, p. 65). Sixty percent of reported altitude deviations worldwide take place in the descent and approach phases of flight, with Margison (2014) highlighting a threefold increase in the number of reported altitude deviations from 2011 to 2012. The year 2012 marked the beginning of the NextGen mid phase where many of the procedures developed in the short phase (2004-2012) were deployed for operational use. This is significant because the OPD arrival is made up of the descent and approach phases of flight and is designed to be an automated link between en route and terminal airspace (EUROCONTROL, 2017; FAA, 2017).

An air traffic control intervention is defined as “...airspeed, altitude, or heading instructions issued to the aircraft by air traffic control (ATC) that remove it from or modify the published OPD procedure in some manner" and includes assigning airspeeds, altitudes, or headings different from the published procedure or instructions issued in error (Lyle, 2020, p. 83; Flight Safety Foundation, 2000). Air traffic control intervention on an OPD is generally an instruction requiring the flight crew to remove the aircraft from fully automated flight and downgrade to a reduced level of automation or manual control to comply with ATC instructions. This interrupts the flight management computer (FMC) calculated descent path which provides compliance with published procedure airspeed and altitude restrictions. While the aircraft is not on the published arrival procedure, the FMC does not have a known navigation point and altitude restriction on which to base vertical path calculations and evaluate compliance with those 
restrictions (General Electric, 2010). If the aircraft is subsequently cleared to rejoin the published procedure, the FMC once again has lateral and vertical navigation information and calculates a new descent profile. At this point the crew may not correctly resume automated flight, there may be an error by the crew or ATC in communicating or understanding the clearance, or compliance with the clearance may exceed the performance capabilities of the aircraft (Lyle, 2020). This is the point where an altitude deviation may occur.

Altitude Deviation as a Leading Safety Indicator. Breaking down a complex system such as the National Airspace System (NAS) to identify potential safety issues involves the use of benchmarks portraying system status. Leading safety indicators are system-specific signals that may show the presence of "... warning signs..." for accident causation and "...directly correlate to future performance..." of the system (Britton, 2019, p. 1). Leading indicators are proactive indices that may point to a need for action when system values exceed certain thresholds, suggesting that system safety has "migrated" to an unacceptable level of risk over time (Leveson, 2015). Further analysis of a leading indicator may also provide insight as to why the system has moved to this increased risk level. Using these definitions, the argument can be made that the altitude deviation rate increasing by a magnitude of three in 2012 and failing to return to historic norms during subsequent years suggest a difference between system design and system use. In the context of the potentially catastrophic consequences of a single mid-air collision where altitude deviation is a factor, however remote, altitude deviation is a leading safety indicator in the NAS, as described by Leveson (2015) and congruent with the proactive safety approach of the NextGen Integrated Plan (DOT, 2004; Lyle, 2020).

\section{Background}

The Next Generation Integrated Plan is the theoretical construct on which the NextGen airspace modernization program is based (DOT, 2004). Concepts developed during the NextGen short phase (2004-2012) were to be deployed in an integrated fashion during the mid-phase (2012-2020) producing operational data for system improvement and refinement during the long phase, 2020 and beyond (DOT, 2004; Houston, 2017). The FAA's updates and progress reports on NextGen implementation document that the intended integrated deployment has not taken place to date; and, in one instance reported an initiative to target the congested and airspaceconstrained northeastern U.S. for a more integrated use of automated NextGen procedures (FAA, 2016, 2018, 2019). More recent FAA performance initiatives have attempted to shift the burden of improving NAS metrics to the airlines without addressing improvements to the underlying system infrastructure and operational philosophy which would enable the airlines to meet these goals (FAA, 2020). The continued presence of altitude deviations reported on OPD arrivals and the failure of altitude deviation rates to return to historic baseline values portray a system that is not being used in its integrated form as it was designed, and consistent with earlier literature arguing that concurrent use of automated and manual procedures would not be compatible (Lyle, 2020).

Literature pre-dating the publication of The NextGen Integrated Plan in 2004 addressed the incompatibility of using manual and automated procedures together (Billings, 1997). Billings (1997) cautioned against a system that allows for concurrent manual and automated control because the operator may not have the ability to determine which mode is in use and 
could circumvent safety features which were designed into the system. Murdoch, Barmore, Baxley, Abbott and Capron(2009) was a seminal study early in the NextGen short phase that reported increased delivery accuracy at the runway threshold by using automated spacing techniques, and aircraft that failed to meet the target spacing parameters did so due to a manual ATC intervention. The findings of Dao et al. (2010) were consistent with those of Murdoch et al. (2009) and reported that "...findings show that current interval management systems perform better at higher levels of automation where there is low human intervention" (p. 25). In their study using the automated capability required time of arrival (RTA) to meld en route and terminal arrival schedules, Hayashi, Coppenbarger, Sweet, Nagle and Dyer (2011) reported a $97 \%$ increase in delivery accuracy at the terminal area boundary using RTA when compared to manual techniques. This accuracy increases predictability and reduces uncertainty in the traffic flow, maintains safeguards designed into the automated arrival procedure, and allows for safe reduction in aircraft separation. Reduced separation enables the expansion of airport and airspace capacity.

\section{Method}

Theoretical Approach. The exploratory-sequential, mixed-methods approach of qualitative-quantitative- interpretation was used to explore how air traffic control intervention effects altitude deviations on OPD arrivals (Creswell, 2014). Archival data for this study came from two sources: aviation safety reports from the NASA Aviation Safety Reporting System (ASRS) and aviation safety reports from the Aviation Safety Action Program (ASAP) of a U.S. major airline for the study period January 1, 2012 to January 1, 2018. Each of these reports contained textual narratives describing the altitude deviation being reported that enabled the identification of a main theme related to the event. The theme from each narrative was then coded into one of eight independent variables producing numeric values for statistical analysis, the process of quantification described by Landrum and Garza (2015). Correlation analysis was then used to reveal relationships and patterns present in the data that describe the effect of air traffic control intervention on altitude deviations reported on optimized profile descent arrival procedures. "The exploration of these patterns and relationships indicated the use of a mixedmethods approach with its increased depth of understanding and analytic density" (Fielding, 2012; Flick, 2007; Lyle, 2020, p. 69). It is the relationships between variables and the ability to determine effect sizes rather than specific causal factors that is the focus of this study and its methodological approach (Lyle, 2020).

The nature of ASRS and ASAP being voluntary aviation safety reports introduced bias into the data, most notably self-reporting bias which includes recall bias (Althubaiti, 2016). Additionally, the confidential nature of the reports due to the reporter protections of these programs made reliability and validity testing difficult, even though the data was collected through each program's standardized reporting method. The use of a mixed-methods study addressed bias as well as reliability and validity issues through triangulation, providing a decrease in bias and a cogent option for validation (Kennedy, 2009; Turner, 2016). ASRS limitations specifically state that ASRS data represent the lower boundary of reliability rather than a precise measure, and results reported in this study will document a lower boundary of reliability (NASA, 1996). ASAP data is subject to many of the same limitations and results and will also represent a lower boundary of reliability. 
Variables. Eight independent variables were present throughout the literature as main themes related to altitude deviations reported on OPD arrivals. They were: air traffic control intervention, aircrew error, collision avoidance, communication error, equipment malfunction or limitation, other, terrain avoidance, and weather (Buono, 2014; Flight Safety Foundation, 2017; IATA, 2017; Margison, 2014). These are nominal variables with no intensity or scalar component but do represent a primary theme embedded in the aviation safety report narrative.

Qualitative Analysis. Narrative analysis comprised the qualitative portion of the study. Each aviation safety report was screened to ensure that it met study criteria: occurred within the study period in the U.S. NAS, altitude deviation reported, OPD arrival procedure, and FAR 121 air carrier (Lyle, 2020). Aviation safety reports are the direct observation of the person involved in the event being reported. ASRS and ASAP data are well-suited to the performance, structural, and literary narrative analytical method of Rogan and de Kock (2005) by “...solicitation of specific narrator experiences...” (p. 632), “...probed for a deeper interpretation of meaning through rich evidential detail..." (p. 638), and, “...evidence for interpretation in the stories was also sought by examining the literary convention of plot... and the narrator's connecting logic of the sequence of events" (pp. 641-642). Using this method, the primary theme from each report was coded into one of the eight independent variables using NVivo (Version 12) qualitative analysis software, and the narrative text catalogued in the appropriate variable node to support variable selection. This process was repeated for each report in the initial ASRS and ASAP datasets which produced 393 ASRS cases and 1791 ASAP cases that met study criteria for quantitative analysis (Lyle, 2020). Both sets of data were procured in chronological order from January 1, 2012 to January 1, 2018 and were divided into four 18-month time periods. Once each case was coded into its appropriate independent variable, this allowed for frequency analysis of the total number of cases contained in each variable and the evaluation of frequency changes over time.

Quantitative Analysis. To determine effect sizes and significant relationships between variables, correlation analysis was performed using SPSS 24 statistical software. As stated earlier, the purpose of this study is to determine the effect of air traffic control intervention on altitude deviations on OPD arrival procedures, not causal factors. Therefore, it is important to set statistical significance and statistical power at appropriate levels to detect significant correlations and minimize both Type I and Type II error rates (Field, 2013). A statistical significance level of $p<.01$ and statistical power of .80 were used in this study with both ASRS and ASAP samples $(N=60)$ being large enough to detect large effect sizes (Cohen, 1992). The correlation analysis used a bootstrap sample size of 1,000. Cronbach alpha was not used as an exact measure of reliability in this study due to the archival and confidential nature of the data, but as a "...lower boundary measurement of the presence of variable correlations within the data" (Lyle, 2020, p. 92). A complete description of the determination of these values may be found in Lyle (2020, pp. 89- 92).

Interpretation. To determine how air traffic control intervention effects altitude deviations on OPD arrival procedures, effect sizes were computed for interpretation. This was done by squaring the Pearson's correlation coefficient $(r)$ for two variables calculated in the correlation analysis to produce the coefficient of determination $\left(R^{2}\right)$ or effect size (Field, 2013). 
Effect size "...is a measure of the amount of variance in one variable that is shared by the other" or the strength of the relationship between two variables (Field, 2013, p. 276). Effect size $\left(R^{2}\right)$ was interpreted as small $=.10$, medium $=.30$, and large $=.50($ Cohen, 1992; Field, 2013).

\section{Results}

Qualitative Results. Air traffic control intervention effects altitude deviations reported on OPD arrival procedures primarily in the areas of aircrew error, communication error, and equipment malfunction or limitation. Qualitative frequencies from the ASRS and ASAP narrative analysis are shown by 18-month period labeled Alt Dev1, Alt Dev 2, etc. in Tables 1 and 2 (Lyle, 2020). Frequencies are the number of cases coded into each variable for each period.

Table 1

ASRS Altitude Deviation Frequencies

\begin{tabular}{|c|c|c|c|c|c|}
\hline Variable/Time Period & Alt Dev1 & Alt Dev 2 & Alt Dev 3 & Alt Dev 4 & Total \\
\hline Air Traffic Control Intervention & 33 & 29 & 25 & 24 & 111 \\
\hline Aircrew Error & 24 & 20 & 11 & 13 & 68 \\
\hline Collision Avoidance & 5 & 6 & 3 & 1 & 15 \\
\hline Communication Error & 28 & 13 & 6 & 8 & 55 \\
\hline Equipment Malfunction or Limitation & 33 & 22 & 17 & 24 & 96 \\
\hline Other & 1 & 1 & 1 & 1 & 4 \\
\hline Terrain Avoidance & 0 & 2 & 0 & 1 & 3 \\
\hline Weather & 12 & 5 & 12 & 12 & 41 \\
\hline Total & 136 & 98 & 75 & 84 & 393 \\
\hline
\end{tabular}

Table 2

ASAP Altitude Deviation Frequencies

\begin{tabular}{|c|c|c|c|c|c|}
\hline Variable/Time Period & Alt Dev 1 & Alt Dev 2 & Alt Dev 3 & Alt Dev 4 & Total \\
\hline Air Traffic Control Intervention & 206 & 233 & 202 & 126 & 767 \\
\hline Aircrew Error & 138 & 94 & 121 & 84 & 437 \\
\hline Collision Avoidance & 15 & 5 & 5 & 2 & 27 \\
\hline Communication Error & 49 & 68 & 67 & 40 & 224 \\
\hline Equipment Malfunction or & 53 & 68 & 54 & 39 & 214 \\
\hline \multicolumn{6}{|l|}{ Limitation } \\
\hline Other & 2 & 6 & 12 & 6 & 26 \\
\hline Terrain Avoidance & 0 & 1 & 0 & 0 & 1 \\
\hline Weather & 21 & 24 & 31 & 19 & 95 \\
\hline Total & 484 & 499 & 492 & 316 & 1791 \\
\hline
\end{tabular}

Quantitative Results. The purpose of this study was to determine how air traffic control intervention effected altitude deviations on OPD arrival procedures. Effect size was determined by squaring the correlation coefficient calculated by correlation analysis for each significant correlation. This produced the coefficient of determination, $R^{2}$, or effect size of each significant correlation (Field, 2013). Effect sizes for significant correlations in the ASRS and ASAP data are shown in Tables 3 and 4 (Lyle, 2020). Large effects are $>.50$. 
Table 3

Effect Sizes of Significant ASRS Data Correlations

\begin{tabular}{ll}
\multicolumn{1}{c}{ Correlation } & $\boldsymbol{R}^{\mathbf{2}}$ \\
\hline Air Traffic Control Intervention / Aircrew Error & .92 \\
Air Traffic Control Intervention / Communication Error & .90 \\
Air Traffic Control Intervention / Equipment Malfunction or Limitation & .61 \\
Aircrew Error / Collision Avoidance & .58 \\
Aircrew Error / Communication Error & .86 \\
Aircrew Error / Equipment Malfunction or Limitation & .67 \\
Collision Avoidance / Communication Error & .32 \\
Collision Avoidance / Weather & .48 \\
Communication Error / Equipment Malfunction or Limitation & .85 \\
Terrain Avoidance / Weather & .72 \\
\hline
\end{tabular}

Table 4

Effect Sizes of Significant ASAP Data Correlations

\begin{tabular}{ll}
\hline \multicolumn{1}{c}{ Correlation } & $\boldsymbol{R}^{\mathbf{2}}$ \\
\hline Air Traffic Control Intervention / Aircrew Error & .21 \\
Air Traffic Control Intervention / Collision Avoidance & .18 \\
Air Traffic Control Intervention / Communication Error & .67 \\
Air Traffic Control Intervention / Equipment Malfunction or Limitation & .90 \\
Air Traffic Control Intervention / Terrain Avoidance & .36 \\
Air Traffic Control Intervention / Weather & .24 \\
Aircrew Error / Collision Avoidance & .74 \\
Aircrew Error / Terrain Avoidance & .17 \\
Collision Avoidance / Other & .38 \\
Communication Error / Equipment Malfunction or Limitation & .72 \\
Communication Error / Other & .30 \\
Communication Error / Terrain Avoidance & .34 \\
Communication Error / Weather & .67 \\
Equipment Malfunction or Limitation / Terrain Avoidance & .67 \\
Equipment Malfunction or Limitation / Weather & .17 \\
Other / Weather & .71 \\
\hline
\end{tabular}

The presence of these large effects suggest that altitude deviation is a leading safety indicator in the U.S. National Airspace System. As such, large effects suggest that the U.S. National Airspace System and NextGen specifically are not being used as they were designed. Aircrew error was previously mentioned as a significant correlation with air traffic control intervention. Taking this effect one step further, there exists a large effect between aircrew error and collision avoidance in both the ASRS (.58) and ASAP (.74) data. The data indicate a positive correlation between air traffic control intervention and aircrew error, an increase in one produced an increase in the other. These data also show a positive correlation between aircrew error and collision avoidance. The complex coupled nature of air traffic management in the U.S. National Airspace System illustrate how these variables are interrelated, but not necessarily directly. Air traffic control intervention does not correlate directly with collision avoidance in the data but does correlate with it indirectly through aircrew error. These findings are consistent with Billings (1997) who observed that isolating errors in a complex system may be difficult due to the opacity of indirect relationships and the potential of having automated and manual control in use simultaneously. 


\section{Discussion}

The results in Tables 3 and 4 show that air traffic control intervention had some of the largest number of statistically significant correlations at $p<.01$ and some of the largest effect sizes $\left(R^{2}\right)$ for both the ASRS and ASAP data groups (Lyle, 2020). Air traffic control intervention had large effects with communication error and equipment malfunction or limitation in both data sets, a large effect with aircrew error in the ASRS data, and small effect with aircrew error in the ASAP data. These effects do not occur in isolation but describe a sequence of events that lead to an altitude deviation with potential safety-related consequences. An example of this would be the large effect between air traffic control intervention and equipment malfunction or limitation. Air traffic control issues a clearance that takes the aircraft off the charted OPD arrival. The aircraft is then issued a clearance to resume the arrival and comply with an altitude restriction that exceeds the performance capabilities of the automated flight management system resulting in an altitude deviation. This example also illustrates the disruption caused by manual intervention in an automated system that had previously calculated aircraft performance requirements that met procedural restrictions.

It is precisely these types of interrelationships that give altitude deviation its value as a leading safety indicator. The frequency data in Tables 1 and 2 do indicate a declining trend in the number of cases citing air traffic control intervention as a factor in a reported altitude deviation. However, Tables 1 and 2 continue to show air traffic control intervention as the leading factor cited in an altitude deviation report during the last 18 months of the study period (Alt Dev 4). Correlation analysis by its definition does not identify a causal factor, but the method does identify relationships and their strength in the data. The continued presence of these frequencies in Tables 1 and 2 and the large effects in Tables 3 and 4 suggest that air traffic control intervention is still affecting altitude deviations. Leading safety indicators identify areas of concern before an accident happens, and this information can be used to gain a better understanding of contributing factors even if they are not readily apparent. A more complete understanding of the altitude deviation event and the interplay of contributing factors provide a more comprehensive view of how the system is being used, and insight into the effectiveness of design safety measures. Should these safeguards be inadequate to prevent a migration from the desired level of safety within the system, mitigating procedures may be introduced or enhanced to return the system to the acceptable safety level for which it was designed (Leveson 2015). In this context, the data show that the confluence of automated and manual air traffic control procedures creates a locus for an altitude deviation, and mitigating action is warranted to reduce or eliminate this practice. The failure of the altitude deviation rate to return to historic norms and the continued presence of significant large effects between air traffic control intervention and other study variables indicate that this practice is still taking place and potentially compromising aviation safety.

\section{Conclusions}

Air traffic control intervention directly effects altitude deviations reported on OPD arrival procedures primarily in the areas of aircrew error, communication error, and equipment malfunction or limitation. Altitude deviation is a leading safety indicator for the U.S. National Airspace System, and it is suggesting that the system is not being used in the integrated manner 
outlined by the NextGen Integrated Plan, and that the system safety level is being compromised by the continued concurrent use of automated and manual procedures. The literature argued that these two methods of control would not be compatible when used together, and the effect sizes from these data suggest that this is indeed the case. This contradicts the proactive safety thread of the NextGen Integrated Plan in that built-in automated safety design is bypassed when manual control is introduced, and there were no provisions or safety planning in the Integrated Plan that allowed for combined use of automated and manual procedures. Taking the systems theory view, this hybrid automated/manual control environment has not been vetted for potential safety conflicts or examined in-depth to explore unintended negative safety-related outcomes. 


\section{References}

Althubaiti, A. (2016). Information bias in health research: Definition, pitfalls, and adjustment methods. Journal of Multidisciplinary Healthcare, 9, 211-217. DOI:

10.2147/JMDH.S104807

Billings, C. (1997). Aviation automation: The search for a human-centered approach. Boca Raton, FL: CRC Publishing

Britton, T. (2019). What are leading safety indicators in aviation SMS? Retrieved from http://aviationsafetyblog.asms-pro.com/blog/what-are-leading-indicators-in-aviation-smsprograms

Buono, G. (2014). Altitude deviations: STEADES in-depth analysis. IATA Airborne Conflict Safety Forum, Brussels, Belgium, June 2014. Retrieved from www.skybrary.aero/bookshelf/content/bookdetails.php?bookld=2828

Cohen, J. (1992). Power primer. Psychological Bulletin, 112, 155-159. Retrieved from www.researchgate.net

Conklin and de Decker (2021). CO2 emissions; causes and cost. Retrieved from https://www.conklindd.com/t-Articlesco2emissionscausesandcosts.aspx\#: :text=Let's start with a basic, over 22 pounds of $\mathrm{CO} 2$

Creswell, J. (2014). Research design: Qualitative, quantitative, and mixed methods approaches. Thousand Oaks, CA: SAGE Publications

Dao, A., Lachter, J., Battiste, V., Brandt, S., Vu, K., Strybel, T., Ho, N., Martin, P., \& Johnson, W. (2010). Automated spacing support tools for interval management operations during continuous descent approaches. Proceedings of the Human Factors and Ergonomics Society, 54th Annual Meeting, September 27-October 1, 2010, San Francisco, CA, USA. Retrieved from www.human-factors.arc.nasa.gov.

EUROCONTROL (2017). Continuous descent. Retrieved from www.skybrary.aero/index.php/Continuous_Descent

Federal Aviation Administration [FAA] (2016). Nextgen priorities joint implementation plan executive report, rolling plan 2017-2019. Retrieved from www.faa.gov

Federal Aviation Administration [FAA] (2017). Aeronautical information manual: Change 3. Retrieved from www.faa.gov

Federal Aviation Administration [FAA] $(2018,2019)$. Air traffic by the numbers. Retrieved from www.faa.gov/air_traffic_by_the_numbers 
Federal Aviation Administration [FAA] (2020). FAA-performance initiatives. Retrieved from www.swalife.com

Fielding, N. (2012). Triangulation and mixed methods design: Data integration with new research technologies. Journal of Mixed Methods Research, DOI: $10.1177 / 1558689812437101$

Flick, U. (2007). Designing qualitative research. London, England: SAGE Publications

Flight Safety Foundation (2000). Approach and landing accident reduction toolkit: FSF Briefing Note 3.2-Altitude Deviations. Retrieved from www.flightsafety.org

Flight Safety Foundation (2017). Level bust. Retrieved from www.flightsafety.org

Field, A. (2013). Discovering statistics using IBM SPSS statistics (4th ed.). London, England: SAGE Publications

General Electric [GE] (2010). FMCS guide: FMC update 10.8A. Retrieved from www.swalife.com

Hayashi, M., Coppenbarger, R., Sweet, D., Nagle, G., \& Dyer, G. (2011). Impacts of intermediate cruise-altitude advisory for conflict-free continuous-descent arrival. American Institute of Aeronautics and Astronautics, AIAA Guidance, Navigation, and Control Conference, August 8-11, 2011, Portland, OR. Retrieved from www.aviationsystemsdivision.arc.nasa.gov/publications/2011/AIAA-2011-6216

Houston, S. (2017). NextGen in a nutshell. Retrieved from www.thebalance.com/282561

International Air Transport Association [IATA] (2017). STEADES air traffic services (ATS) analysis. Retrieved from www.iata.org/docx/iata-safety-report-2016-2.pdf

Kennedy, P. (2009). How to combine multiple research methods: Practical triangulation. Retrieved from www.johnnyholland.org/2009/08/practical-triangulation

Landrum, B., \& Garza, G. (2015). Mending fences: Defining the domains and approaches of quantitative and qualitative research. Qualitative psychology, 2(2), 199-209. Retrieved from www.dx.doi.org/10.1037/qup0000030

Leveson, N. (2015). A systems approach to risk management through leading safety indicators. Reliability Engineering and System Safety, 136, 17-34. Retrieved from www.elsevier.com/locate/ress 
Lyle, D. (2020). The effect of air traffic control intervention on reported altitude deviations during optimized profile descent arrival procedures (Order No. 28151055). Available from Dissertations \& Theses @ Saint Louis University; ProQuest Dissertations \& Theses Global. (2460673297). Retrieved from https://ezp.slu.edu/login?url=https://wwwproquest-com.ezp.slu.edu/dissertations-theses/effect-air-traffic-control-interventionon/docview/2460673297/se-2?accountid=8065

Margison, G. (2014). Altitude Deviations: STEADES high-level analysis. IATA. Retrieved from www.skybrary.aero

Murdoch, J., Barmore, B., Baxley, B., Abbott, T., \& Capron, W. (2009). Evaluation of an airborne spacing concept to support continuous descent arrival operations. Eighth USA/Europe Air Traffic Management Research and Development Seminar (ATM 2009). Retrieved from www.scholargoogle.com

National Aeronautics and Space Administration [NASA] (1996). ASRS database statistics 1994. Retrieved from www.asrs.arc.nasa.gov/publications/directline/dl8_stat.htm

Rogan, A., \& de Kock, D. (2005). Chronicles from the classroom: Making sense of the methodology and methods of narrative analysis. Qualitative Inquiry, 11, 628-649. DOI: $10.1177 / 1077800405276777$

Southwest Airlines (2018). Southwest corporate fact sheet. Retrieved from www.swmedia.com/pages/corp-factsheet

Turner, J. (2016). Triangulation: A technique to reduce bias and improve validity and reliability. Retrieved from www.performanceexpress.org/2015/11/hpt-research-fit-triangulation-atechnique-to-reduce-bias-and-improve-validity-and-reliability

U.S. Department of Transportation [DOT] (2004). Next generation air transportation system integrated plan. Retrieved from www.dot.gov 